\title{
Nitric oxide - Its importance in swallowing, inflammatory bowel disease and cirrhotic cardiomyopathy
}

\author{
ABR Thomson MD FRCPC
}

\begin{abstract}
ARTICLES
Beyak MJ, Xue S, Collman PI, Valdez DT, Diamant NE. Central nervous system nitric oxide induces oropharyngeal swallowing and esophageal peristalsis in the cat. Gastroenterology 2000;119:377-85.
\end{abstract}

Kubes P. Inducible nitric oxide synthase: a little bit of good in all of us. Gut 2000;47:6-9.

McCafferty DM. Peroxynitrite and inflammatory bowel disease. Gut 2000;46:436-9.

Liu H, Ma Z, Lee SS. Contribution of nitric oxide to the pathogenesis of cirrhotic cardiomyopathy in bile duct-ligated rats. Gastroenterology 2000;118:937-44.

$\mathrm{N}$ itric oxide is a neurotransmitter found in the central and peripheral nervous systems. Nitric oxide synthase (NOS) is localized in the central nervous system, including the nucleus of the solitary tract, nucleus ambiguus and dorsal motor nucleus of the vagus. These are regions that are implicated in the central control of swallowing and esophageal motility. In rats and rabbits, NOS has been shown to be present in the nucleus subcentralis of the nucleus of the solitary tract, and is thought to be responsible for the central programming of the striated muscle component of esophageal peristalsis. Beyak and co-workers from the University of Toronto, Toronto, Ontario provided evidence that the L-arginine-nitric oxide pathway is implicated in the central control of swallowing and esophageal motility. They studied oropharyngeal swallowing as well as esophageal peristalsis, and determined the functional role of brain stem nitric oxide by examining the effects of blockade of central nervous system NOS on swallowing, and on primary and secondary peristalsis. Administering NOS inhibitors intravenously or intracerebroventricularly into the fourth ventricle produced a number of oropharyngeal swallows and induced primary peristalsis in the smooth muscle portion of the esophageal body. NOS reduced the number of oropharyngeal swallows and the incidence of primary peristalsis in both smooth and striated muscle, and reduced the amplitude of peristalsis and smooth muscle contraction. This suggests that nitric oxide is a functional neurotransmitter in the central pattern generator responsible for swallowing and the central control of esophageal peristalsis. Peripherally administered NOS inhibitor can access structures within the blood-brain barrier to affect neuronal activity and physiological function. The central pattern generated for swallowing and esophageal peristalsis is sug- 
gested to be a serial network of linked neurons within the nucleus of the solitary tract and neighbouring reticular formation, and there is likely one subnetwork for the oropharyngeal phase and the other for the esophageal phase of swallowing. The neurosubstances mediating striated and smooth muscle peristalsis may be both anatomically and neurochemically distinct. The role of nitric oxide in the pathogenesis of esophageal motility disorders remains to be established.

Over the past 10 years, more than 30,000 papers on nitric oxide have been published. Surprisingly, nitric oxide may have a beneficial as well as a detrimental effect in, for example, models of inflammation. There are numerous forms of NOS, including the constitutive form (cNOS), the neuronal form (nNOS) and the endothelial (eNOS) form, as well as the inducible form (iNOS). Nitric oxide is synthesized from a guanidine group of L-arginine and can be produced by almost all mammalian cells. eNOS appears to be a homeostatic regulator of numerous essential cardiovascular functions. In the gastrointestinal tract, cNOS regulates epithelial permeability. Inhibition of nitric oxide causes many of the features of intestinal inflammation, including increased neutrophil recruitment, increased oxidative stress, mast cell degranulation, and increased microvascular and epithelial permeability. In the trinitrobenzene sulphonic acid (TNBS) model of colitis, inhibition of NOS by the oral administration of nonspecific inhibitors in the intestine exposed to TNBS provides dramatic protection and restitution in seven days, yet chronic NOS inhibition in the absence of TNBS causes intestinal inflammation. In a timely review of iNOS, Kubes et al proposed that the iNOS-producing cells need to be characterized to determine what role this inhibition may play in health and disease. They suggested that the hypothesis that "...cNOS is good and iNOS is bad is far too simplistic and no longer explains the majority of data generated by the scientific community".

The etiology of the initiation and recurrence of inflammatory bowel disease is unknown, and a number of factors have been proposed, including increased epithelial motility, inappropriate neutrophil infiltration, activation of mast cells and increased concentrations of pro-inflammatory mediator such as cytokines, leukotrienes and reactive oxygen metabolites. Nitric oxide has also received attention as an important player in the pathogenesis of inflammatory bowel disease. Nitric oxide is a weak free radical that can react with superoxide to produce peroxynitrite. McCafferty et al elegantly reviewed the importance of peroxynitrite and inflammatory bowel disease, and proposed that this molecule may play an important role in the physiological and pathophysiological processes contributing to inflammatory bowel disease.

The hyperdynamic circulation seen in patients with cirrhosis may be due to increased vascular tone, whereas cirrhotic cardiomyopathy may be due to impaired cardiac contractile responsiveness to stressful stimuli. The vasodilator nitric oxide is thought to be a possible mediator of the hyperdynamic circulation in cirrhosis, in which increased levels of cytokines stimulate the activity of iNOS to produce excess amounts of nitric oxide. In noncirrhotic humans and in animal models of heart failure, nitric oxide is a negative inotropic and chronotropic agent. In bile ductligated cirrhotic rats, cardiac tumour necrosis factor (TNF)- $\alpha$, iNOS mRNA and protein, cGMP and interleukin-1 (IL-1)- $\beta$, and nitrite/nitrate levels were higher than in controls (Liu et al). The increased cytokinemia in cirrhosis may be due to enteric bacterial translocation through the gut wall, resulting in portal venus bacteremia and endotoxemia. The well known negative inotropic effects of TNF- $\alpha$ and IL-1 $\beta$ are abrogated by $N^{G}$-nitro-L-arginine methyl ester administration, indicating that these effects are mediated by a nitric oxide-dependent pathway, at least in the cirrhotic heart. Nitric oxide inhibits beta-adrenergic receptor-stimulated contractility via a cGMP-mediated inhibition of voltagedependent calcium ion current. The authors propose that the increased nitric oxide production in this animal model may play an important role in the pathogenesis of cirrhotic cardiomyopathy. 


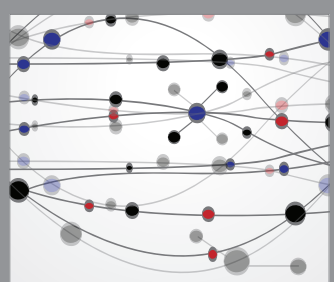

The Scientific World Journal
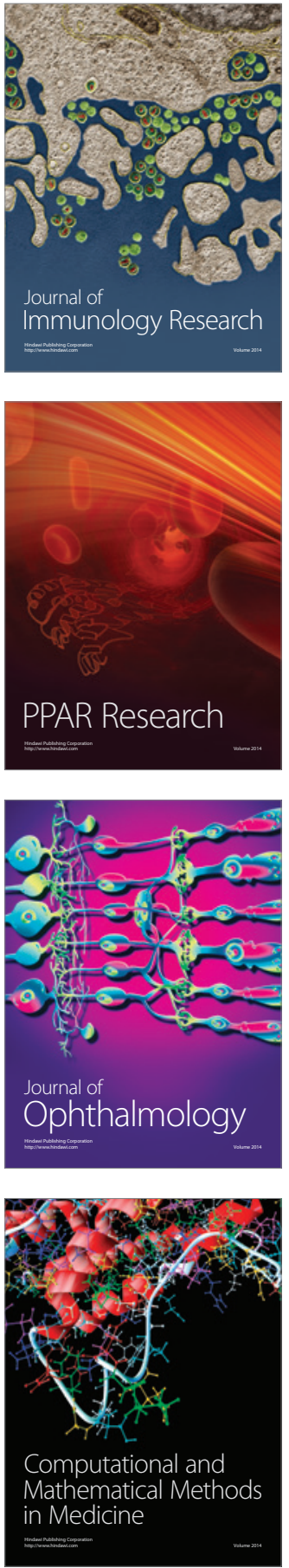

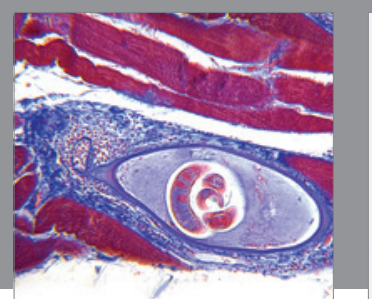

Gastroenterology Research and Practice

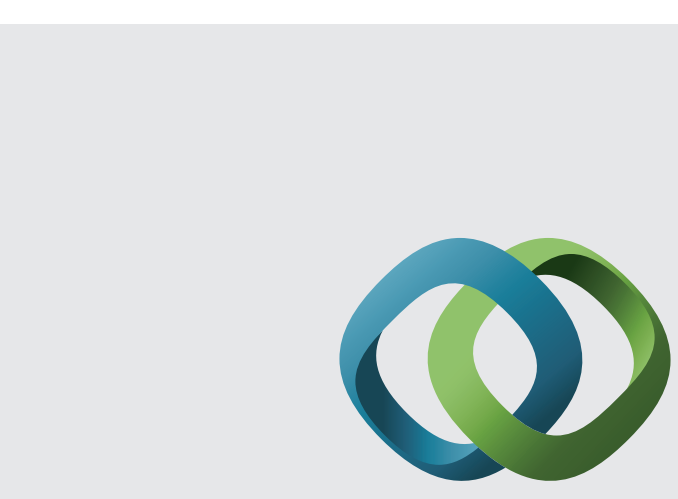

\section{Hindawi}

Submit your manuscripts at

http://www.hindawi.com
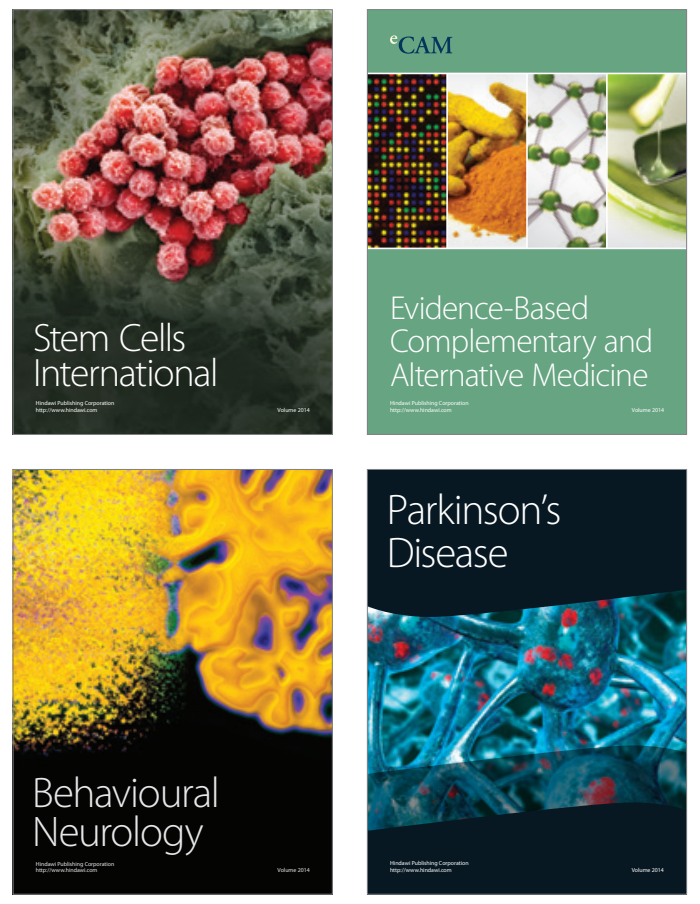
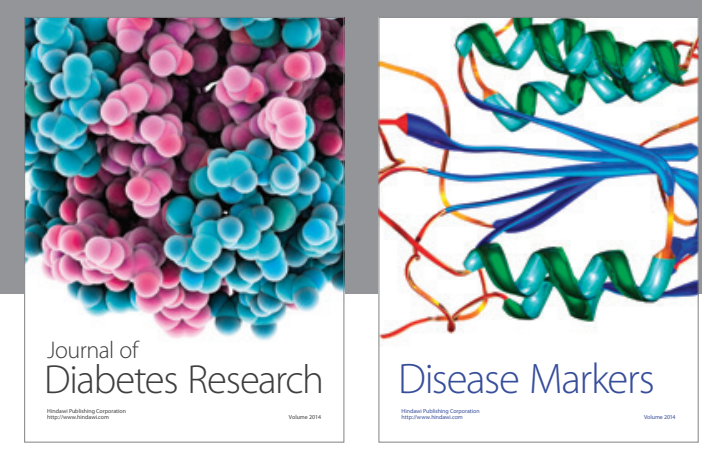

Disease Markers
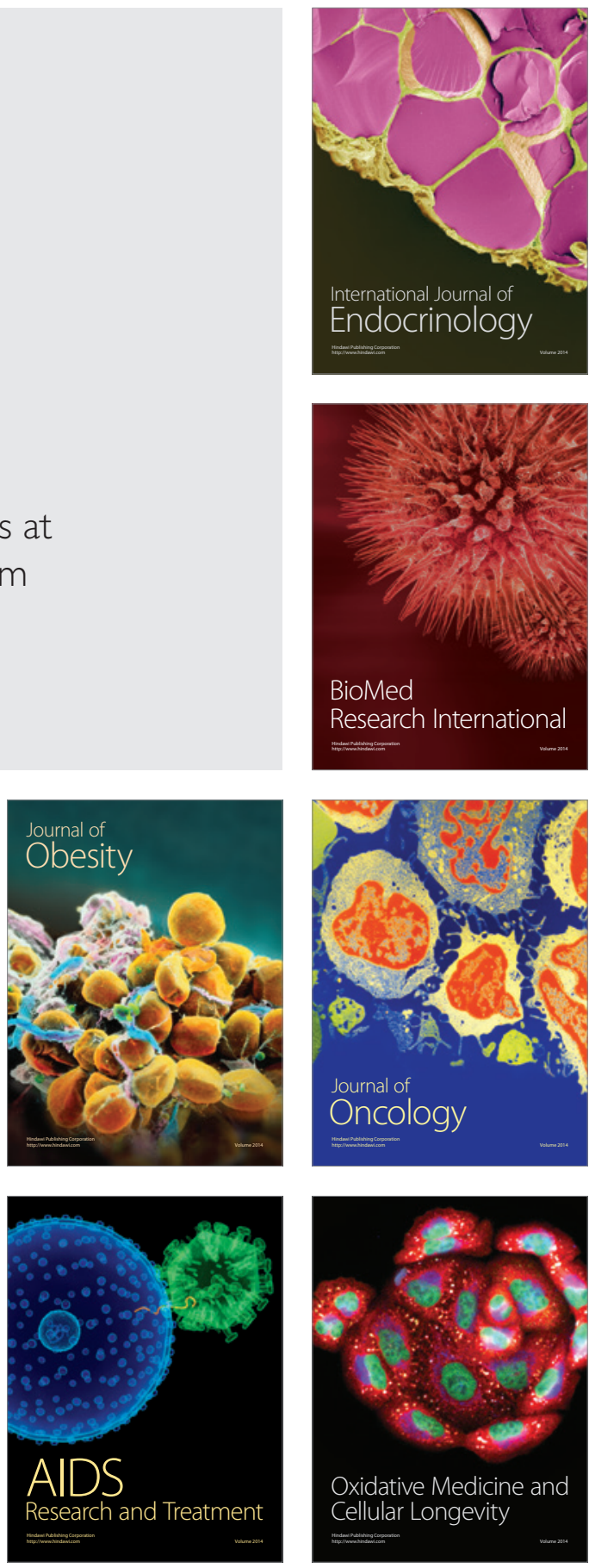